\title{
Fourier-based maximum entropy method in stochastic dynamics
}

\author{
Jorge E. Hurtado ${ }^{\mathrm{a}}$, Alex H. Barbat ${ }^{\mathrm{b}, *}$ \\ ${ }^{a}$ National University of Colombia, Apartado 127, Manizales, Colombia \\ ${ }^{\mathrm{b}}$ Technical University of Catalonia, c/ Gran Capitán, s/n, Module C-1, Barcelona 08034, Spain
}

\begin{abstract}
As the recent research by Trębicki and Sobczyk has demonstrated [1-3] the principle of maximum entropy is a powerful tool for solving stochastic differential equations. In particular, its use in connection with the moment equations generated by the Ito formula provides accurate estimations of the probability density evolution of some oscillators for which conventional methods such as the diverse closure schemes are not applicable. A major computational requirement of the method, however, lies in the need of calculating a large number of multidimensional integrals at each time step-a numerical task for which both accurate and economic algorithms are required. In this paper it is shown that conventional economic integration techniques often lead to numerical collapse of the solution, especially when dealing with higly nonlinear oscillators. A strategy that overcomes this difficulty is proposed. In essence, the integrals are reformulated in terms of multidimensional Fourier transforms, which are solved by an ad hoc FFT algorithm aimed at obtaining only one single "frequency" point. It is demonstrated that the numerical stability and the accuracy of the proposed algorithm are superior to those afforded by other integration schemes. (C) 1998 Elsevier Science Ltd. All rights reserved.
\end{abstract}

Keywords: Stochastic differential equations; Fast Fourier transform; Principle of maximum entropy; Random vibration; Nonlinear systems; Numerical integration

\section{Introduction}

A random vibration problem that can be modelled as Markovian can be regarded as solved if the evolution of the joint probability density function of the implied random variables can be accurately estimated. Since analytical solutions are very rare, especially in the non stationary case, several methods have been developed as alternatives to the easy-to-handle but costly Monte Carlo technique. Methods such as equivalent linearization, stochastic averaging [1] or

\footnotetext{
${ }^{*}$ Corresponding author.
} 
closure schemes [2] provide different levels of statistical information, ranging from the first two moments to estimates of the probability functions. Putting aside those methods that only provide low order stochastic information, one is left with different closure strategies which give estimations of the higher order moments upon which densities and distributions can be inferred. A major disadvantage of most of these techniques, however, is that they are applicable only to polynomial types of nonlinearities in the equations of motion. For other types it is necessary to apply simplifying methods, e.g. the search of equivalent polynomials [3]. On the other hand, interesting proposals such as the use of Taylor moments and cumulants [4] require the knowledge of derivatives of the non linear expression of the restoring forces, with the consequence that discontinuous functions, such as the Heaviside or Dirac ones, spring up at very low orders in some cases of nonlinearity. Finally, attempts for solving the Fokker-PlanckKolmogorov equations have been undertaken in the later years by finite element techniques [5-7]. Given the uncoupled structure of those equations, however, the order of the elements is the same as that of the state vector, so that the method is already quite involved even for the simplest cases.

A more general method for solving the system of differential equations of moments based on the principle of maximum entropy has been proposed recently by Trębicki and Sobczyk [8]. It is an extension to the non stationary case of a previous proposal formulated by the same authors for stationary analyses [9]. At first sight it seems to be of unlimited application with respect to types of non linearities and its accuracy has been demonstrated by several examples in the quoted papers. However, the meth each time step, whose estimation and with the dimensio uieres the com
for efficient. n demonstrated by several examp
aputation of several multidimens
etrically with the order of moments
vector. Thus, for a wider and eco ional integrals at culation of integrals is in order.

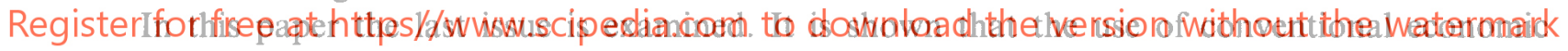
integration strategies, such as Gauss-Legendre quadratures, can lead to numerical unstabilities that collapse the calculation. On the other hand, generally applicable techniques such as Simpson or Romberg integration are quite costly inasmuch as they require an amount of function evaluations too much larger than those needed by Gauss quadratures in the one dimensional case. Since the number of evaluations increases geometrically with the order of the integral this feature render them unuseful for the large number of multidimensional evaluations required by the method of maximum entropy.

In order to overcome these difficulties, a numerical algorithm based on Fourier transforms has been devised. By first transforming the equations of the maximum entropy method into algebraic operations on moments, the calculation of the later is sought by means of the characteristic function at zero frequency. The multidimensional Fast Fourier Transform (denoted as FFT in the sequel) thus formulated is converted into a summation of some one point, one dimensional FFTs which leads to the desired moments. For performing this computation use is made of some features of the transform that make the labour easier to accomplish. In what follows it is shown that the algorithm is more accurate than both Gauss-Legendre and Simpson techniques and as economical as the first one; last but not least, it does not lead to the collapse of the numerical computation of the evolution of the multidimensional density function as the first mentioned technique. 


\section{The method of maximum entropy}

This section is devoted to a brief summary of the application of the maximum entropy method to the solution of systems of stochastic differential equations as formulated in [8]. The theoretical basis on which the method is rooted can be consulted in some standard books on maximum entropy and stochastic differential equations e.g. [10-13].

The principle of maximum entropy, as formulated in the frame of classical theory of probability and mathematical statistics, states that under some constraints (generally given in the form of moments of an $L$-dimensional state vector $\boldsymbol{x}$ or a differential equations of them) the density function $p(\boldsymbol{x})$ that must be chosen is the one that maximizes the entropy functional

$$
H(p)=-\int p(\mathbf{x} ; t) \ln p(\mathbf{x} ; t) \mathrm{d} \mathbf{x}
$$

where the integral spans the entire real space. The constraints (which are more adequately called prior information in the present context) can be given in the form of moments, that is

$$
\mu_{\mathrm{i}}(\mathbf{t})=\int \mathbf{M}_{\mathrm{i}}(\mathbf{x} ; t) \mathrm{d} x=\int m_{\mathrm{i}}(\mathbf{x}) p(\mathbf{x} ; t) \mathrm{d} \mathbf{x}
$$
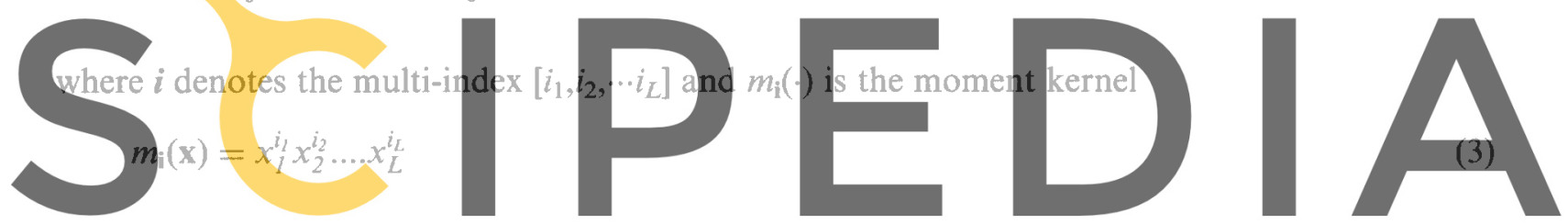

The product $M_{i}(x ; t)=m_{i}(x) p(x ; t)$ will be called "weighted moment kernel" hereinafter. The

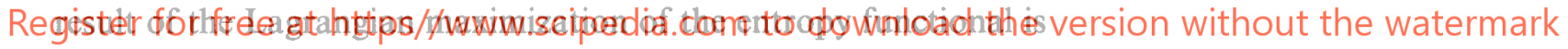

$$
p_{1}(\mathbf{x} ; t)=C_{1}^{-1}(t) q_{1}(\mathbf{x} ; t)=C_{l}^{-1}(t) \exp \left(-\sum_{|\mathbf{k}|=1}^{K} \lambda_{\mathbf{k}} m_{\mathbf{k}}(\mathbf{x})\right)
$$

where $K$ is the highest order of moment considered and $|\boldsymbol{k}|$ is the sum of the elements of the mutiindex vector, i.e.

$$
|\mathbf{k}|=k_{1}+k_{2}+\ldots+k_{L}
$$

In Eq. (4) $C_{1}(t)$ is a normalizing constant, i.e.

$$
C_{1}(t)=\int q_{1}(\mathbf{x} ; t) \mathrm{d} \mathbf{x}
$$

Alternatively, the prior information can be a system of ordinary differential equations (ODE) of moments, which are obtained by application of the Ito formula and the expectation operator to a system of stochastic differential equations (SDE) describing the dynamics of the system. In short, the ODE system can be expressed as 


$$
\dot{\mu}_{\mathbf{i}}(t)=\gamma_{\mathbf{i}}(\mathbf{x})
$$

i.e.

$$
\frac{\mathrm{dE}\left[m_{\mathrm{i}}(\mathbf{x})\right]}{\mathrm{d} t}=\mathrm{E}\left[g_{\mathrm{i}}(\mathbf{x})\right]
$$

in which

$$
g_{\mathbf{i}}(\mathbf{x})=\sum_{j=1}^{L} f_{j} \frac{\partial m_{\mathbf{i}}(\mathbf{x})}{\partial x_{j}}+\frac{1}{2} \sum_{l=l}^{L} \sum_{j, k=1}^{L} \sigma_{j l}(\mathbf{x}) \sigma_{k l}(\mathbf{x}) \frac{\partial^{2} m_{\mathbf{i}}(\mathbf{x})}{\partial x_{j} \partial x_{k}}
$$

where $m_{\mathbf{i}}(\mathbf{x})$ and $\sigma_{\mathrm{jl}}(\mathbf{x})$ are the elements of the drift and diffusion matrices, respectively $[8,13]$. Note that Greek letters are employed here to indicate expected values, while latin ones denote the corresponding kernels. By taking the ODE system as prior information, the maximization of entropy gives the following estimate of the probability density function:

$$
p_{2}(\mathbf{x} ; t)=C_{2}^{-1}(t) q_{2}(\mathbf{x} ; t)=C_{2}^{-1}(t) \exp \left(-\sum_{|\mathbf{k}|=1}^{K} \lambda_{k} g_{k}(\mathbf{x})\right)
$$
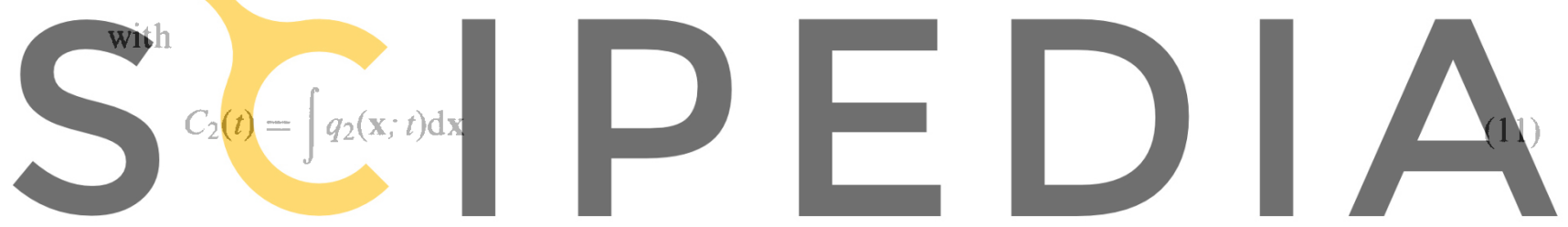

Observing the dual definition of the functions $\gamma_{\mathrm{i}}(\mathbf{x})$, any of the above interpretations of the

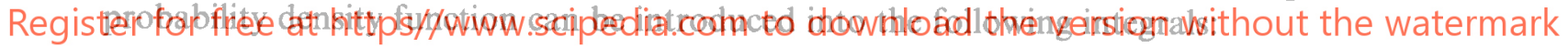

$$
\begin{aligned}
& \gamma_{\mathbf{i}}(\mathbf{x})=\int m_{\mathbf{i}}(\mathbf{x}) \frac{\partial p_{a}(\mathrm{x} ; t)}{\partial t} \mathrm{~d} \mathbf{x} \\
& \gamma_{\mathbf{i}}(\mathbf{x})=C_{a}^{-1}(t) \int g_{\mathbf{i}}(\mathbf{x}) q_{a}(\mathbf{x} ; t) \mathrm{d} \mathbf{x}
\end{aligned}
$$

where the subindex $a$ states for 1 or 2 . If the first interpretation is followed, the evolution of the Lagrange coefficient is given by

$$
\sum_{|\mathbf{k}|=l}^{K}\left[R_{\mathbf{i}}(t) R_{\mathbf{k}}(t)-C_{1}(t) Q_{\mathbf{i k}}(t)\right] \dot{\lambda}_{\mathbf{k}}(t)=C_{1}(t) P_{\mathbf{i}}(t)
$$

where the superimposed dot means time derivative and

$$
R_{\mathbf{i}}(t)=\int m_{\mathbf{i}}(\mathbf{x}) q_{I}(\mathbf{x} ; t) \mathrm{d} \mathbf{x}
$$




$$
\begin{aligned}
& P_{\mathrm{i}}(t)=\int g_{\mathrm{i}}(\mathbf{x}) q_{l}(\mathbf{x} ; t) \mathrm{d} \mathbf{x} \\
& Q_{\mathrm{ik}}(t)=\int m_{\mathbf{i}}(\mathbf{x}) m_{\mathbf{k}}(\mathbf{x}) q_{l}(\mathbf{x} ; t) \mathrm{d} \mathbf{x}
\end{aligned}
$$

Under the second interpretation the corresponding system of algebraic equations is

$$
\sum_{|\mathbf{k}|=l}^{K}\left[\bar{P}_{\mathbf{i}}(t) \bar{R}_{\mathbf{k}}(t)-C_{2}(t) \bar{Q}_{\mathbf{i k}}(t)\right] \dot{\lambda}_{\mathbf{k}}(t)=C_{2}(t) \bar{P}_{\mathbf{i}}(t)
$$

with

$$
\begin{aligned}
& \bar{R}_{i}(t)=\int m_{\mathrm{i}}(\mathbf{x}) q_{2}(\mathbf{x} ; t) \mathrm{d} \mathbf{x} \\
& \bar{P}_{\mathrm{i}}(t)=\int g_{\mathrm{i}}(\mathbf{x}) q_{2}(\mathbf{x} ; t) \mathrm{d} \mathbf{x}
\end{aligned}
$$

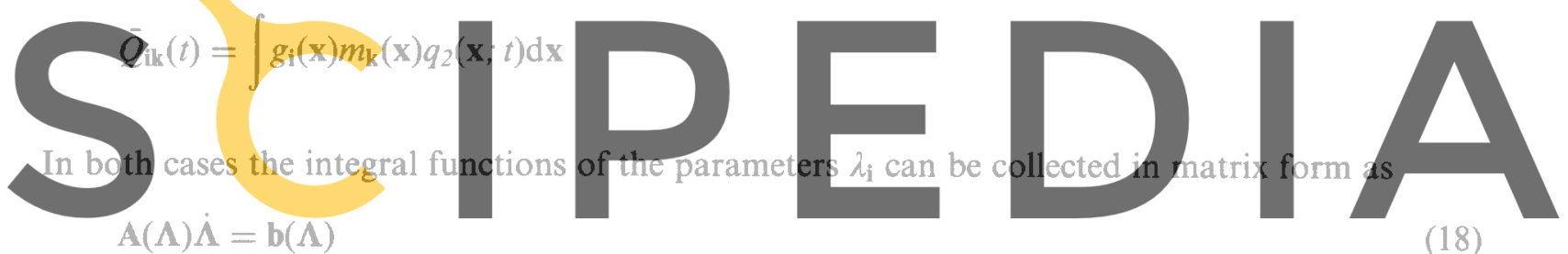

$$
\mathbf{A}(\boldsymbol{\Lambda}) \dot{\Lambda}=\mathbf{b}(\boldsymbol{\Lambda})
$$

(18)

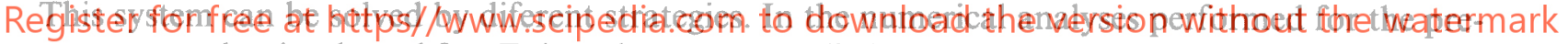
sent paper, the simple and fast Euler scheme was applied as suggested in [8]. It is important to say that the use of more refined numerical methods for solving this ODE system (such as Runge-Kutta or Adams-Moulton) will largely increase the computational labour due to the need of calculating the integrals (15) or (17) and solving the system of algebraic Eq. (18) at each intermediate step.

The first interpretation of the maximum entropy density will be preferred in the present paper due to the following reasons: (a), it is easier to start than the second one, because the initial condition of the density, which is usually a Dirac function corresponding to a deterministic initial situation, can be modelled by a slim Gaussian function from which the initial values of the parameters $\lambda$ in Eq. (4) can be easily obtained. This is not always the case when applying the other method; (b), the terms $Q_{\mathrm{ik}}$ are symmetric while the $\bar{Q}_{\mathrm{ik}}$ are in general not; also some $Q_{\mathrm{ik}}$ coincide with some $R_{\mathrm{i}}$, so that the amount of integrals that need to be evaluated at each step is smaller in the first method than in the second.

\section{Proposed Fourier approach}

It is well known that if an $N$-dimensional integral is to be evaluated by a discretization of $L$ points, the number of function evaluations is $L^{N}$. Thus, the practical use of the method of 
maximum entropy requieres efficient methods for performing such a large numerical task at each time step.

Besides conventional methods for numerical integration, this labour can be faced by having resort to the characteristic function of the maximum entropy density. Dividing both sides of Eq. (14) by $C_{1}^{2}(t)$ it is easy to see that it can be recast in terms of moments, i.e.

$$
\sum_{|\mathbf{k}|=1}^{K}\left[\mu_{\mathbf{i}}(t) \mu_{\mathbf{k}}(t)-\mu_{\mathbf{i}+\mathbf{k}}(t)\right] \dot{\lambda}_{\mathbf{k}}(t)=\mu_{\mathbf{i}}(t)
$$

In passing, it can be seen from the above equation that if the highest order of moment to be evaluated is $K$, the maximum entropy method uses information of order $2 K$, which explains the good accuracy achieved at low $K$ orders as reported in Ref. [8].

While the moments are defined as integrals of the product of the respective kernels by the density function, they can also be expressed as:

$$
\mu_{\mathbf{i}}=\left(\frac{1}{i}\right)^{|\mathbf{i}|}\left(\frac{\partial^{|i|} \Omega(\theta)}{\partial^{i_{1}} \partial^{i_{2}} \ldots \partial^{i_{L}}}\right)_{\theta=0}
$$

where $i^{2}=-1$ and $\Omega(\theta)$ is the characteristic function defined as the Fourier transform of the
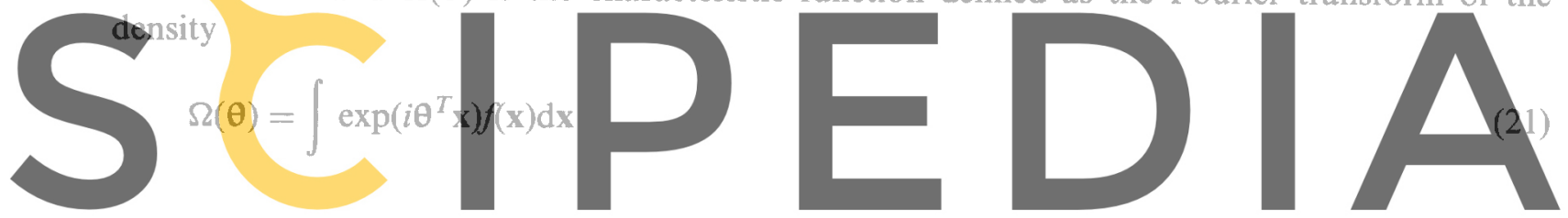

According to this definition the moment of order $i$ can be calculated as the following one point

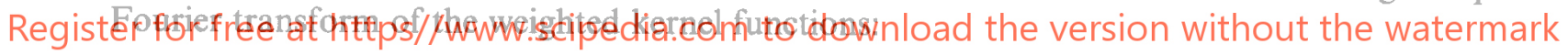

$$
\mu_{\mathbf{i}}=\left(\int \exp \left(i \theta^{T} \mathbf{x}\right) m_{\mathbf{i}}(\mathrm{x}) f(\mathbf{x}) d \mathbf{x}\right)_{\mathbf{\theta}=\mathbf{0}}
$$

It is obvious that any integral spanning the whole real space can be estimated as the real part of the zero frequency value of the Fourier transform, provided the later exists. Hence, from a computational point of view the calculation of the elements of matrix $\boldsymbol{A}$ car be done by taking advantage of some properties of the Fourier transform. Specifically, the computer code written for these analyses takes into account the following facts:

1. The need of calculating one single transform point which corresponds to the zero "frequency". Fig. 1 (adapted from [14]) illustrates by solid lines the operations needed for calculating such single point out of the many needed to compute the discrete Fourier transform

$$
X[k]=\sum_{l=0}^{L-1} x[l] e^{2 \pi i \frac{i k}{L}}
$$

at $L=$ eight locations. The circles represent summation and the functions $W_{L}^{j}$ are the weights $\exp (2 \pi \mathrm{i} j / L)$ applied to the corresponding value. It is evident that the operations indicated by 

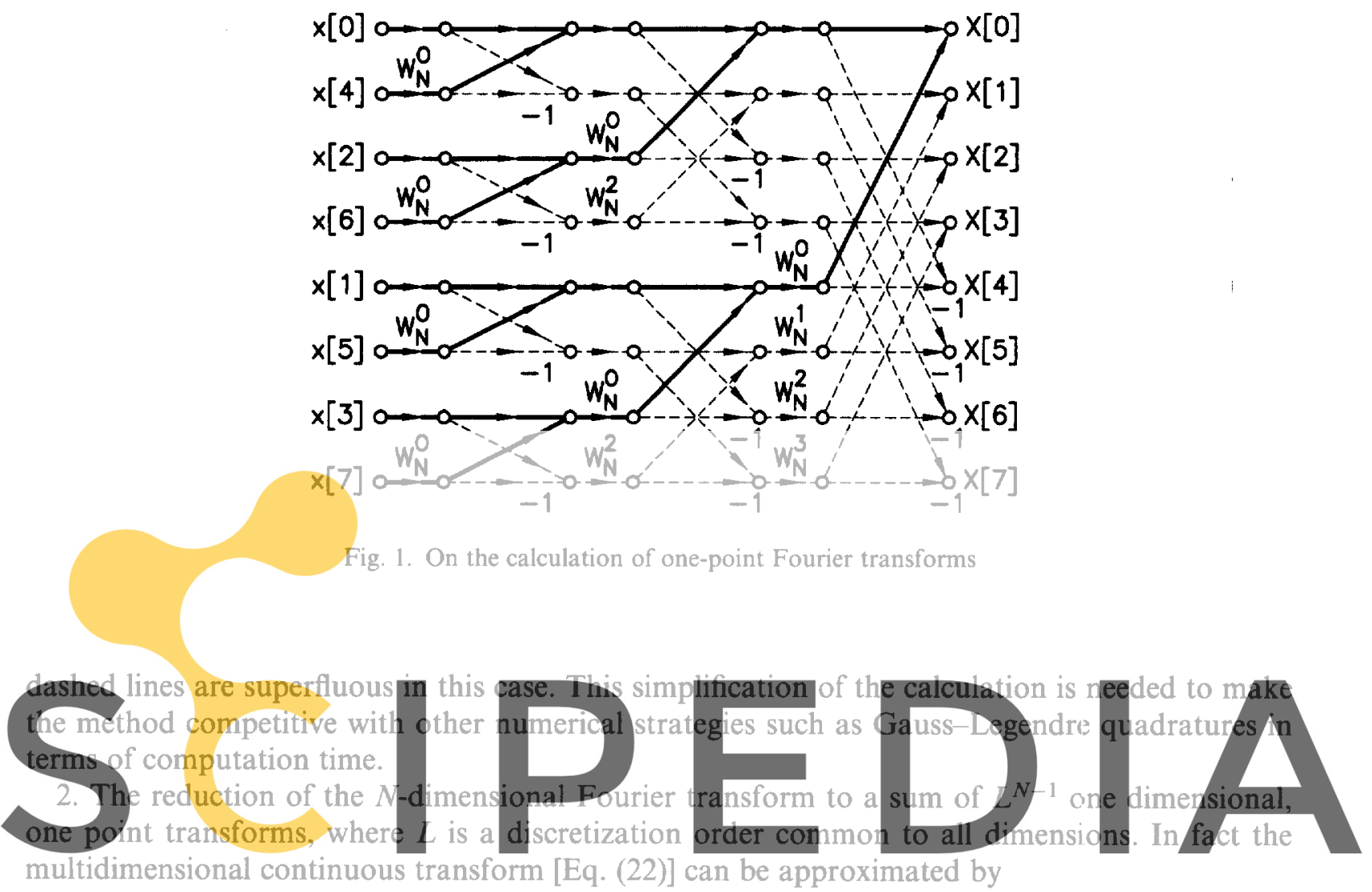

Register for free at https//www.scipedia.com to download the version without the watermark

$$
\sum_{i_{N}=0} \ldots \sum_{l_{1}=0} \exp \left(2 \pi \mathrm{i}\left[\frac{l_{1} k_{1}}{L_{1}}+\ldots+\frac{l_{N} k_{N}}{L_{N}}\right]\right) M_{i}\left(l_{1}, \ldots, l_{N}\right) \cdot \Pi_{n=1}^{N} \Delta_{N}
$$

in which $L_{1}, \ldots, L_{N}$ are the number of discretization points of the weighted moment kernel on each axis, $k_{1}, \ldots, k_{L}$ are the coordinate points of a desired value of the transform, $M_{\mathrm{i}}(\cdot)$ is the value of the $i$ weighted moment kernel evaluated at the point of coordinates $\left(l_{1}, \ldots l_{N}\right)$ and $\Delta_{1}, \ldots, \Delta_{N}$ are the discretization intervals. Since the moment integrals extend over the whole real space, there is no special reason for assigning a different number of discretization points to each axis, so that all of them can be made equal to a certain $L$. Also, by proceeding in this way, the value of all the $k_{n}$, $n=1,2 \ldots . N$ corresponding to the zero frequency happen to be also mutually equal because the null frequency occupies the central position. If its corresponding coordinate is denoted by $k$, the single point multidimensional transform (22) can be estimated by

$$
\sum_{||=0}^{N(L-1)} \exp \left(2 \pi \mathrm{i}\left(\frac{|\mathrm{l}| k}{L}\right)\right) M_{\mathrm{i}}\left(l_{1}, \ldots, l_{N}\right) \cdot \Pi_{n=1}^{N} \Delta_{n}
$$

A simple examination of this equation makes apparent that, by splitting the whole sum into segments according to the index that changes most rapidly, the calculation can be done by summing up $L^{N-1}$ one dimensional, one point transforms. Symbolically, we have 


$$
\operatorname{FFT}(N, k)=\sum_{i=1}^{L^{N-1}} \operatorname{FFT}_{i}(1, k)
$$

Table 1 illustrates the way the splitting must be done in a bidimensional case $(N=2)$ using $L=$ four points so that $L^{N-1}=4$. The elementary single point FFTs are calculated following the paths depicted in Fig. 1.

3. The possibility of calculating two transforms at a time, due to the non-complex nature of the weighted moment kernels, which represents an additional computation time saving. A complex array can be built in such a way that its real and complex parts are filled up by the values of two different weighted kernel functions and the transform will return the corresponding moments simultaneously. This device makes use of the symmetry of the Fourier transform for both purely real and purely imaginary functions.

Fig. 2 shows the shape of a typical Fourier transform of an even moment kernel of a zero mean variable. It can be observed that the zero frequency is the least affected by the so-called aliasing phenomenon, by which the transform values corresponding to frequencies above the critical Nyquist value are folded up and added to the values of the transform corresponding to frequencies lower than it. This means that the transforms can be calculated with a reduced number of points per axis with no serious risk to the accuracy of the algorithm.

Table 2 shows a comparison of the results of the evaluation of the three dimensional kurtosis kernel
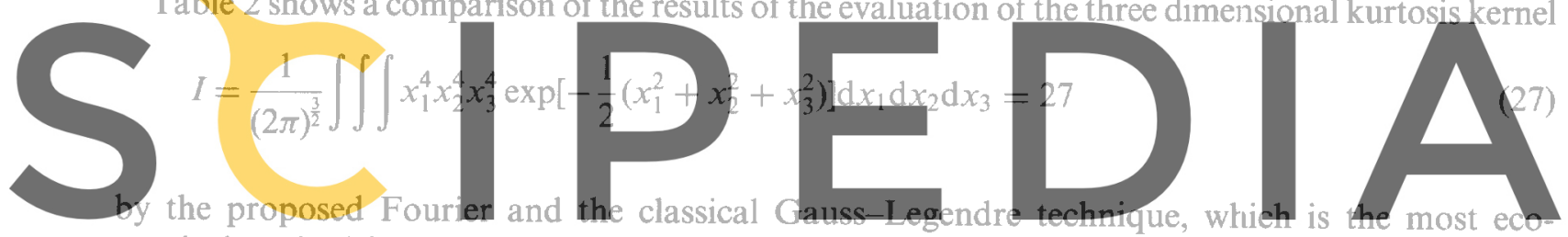

nomical method for this task. It is observed that the Fourier method converges faster to the target Register for free at https//www.scipedia.com to download the version without the watermark

\section{Table 1}

Splitting of a one-point bidimensional Fourier transform

\begin{tabular}{llll}
\hline Single FFT No. & $r_{1}$ & $r_{2}$ & $|\mathbf{r}|$ \\
\hline 1 & 0 & 0 & 0 \\
& 1 & 0 & 1 \\
& 2 & 0 & 2 \\
2 & 3 & 0 & 3 \\
& 0 & 1 & 1 \\
& 1 & 1 & 2 \\
3 & 2 & 1 & 3 \\
& 3 & 1 & 4 \\
& 0 & 2 & 2 \\
4 & 1 & 2 & 3 \\
& 2 & 2 & 4 \\
& 3 & 2 & 5 \\
& 0 & 3 & 3 \\
& 1 & 3 & 4 \\
\end{tabular}




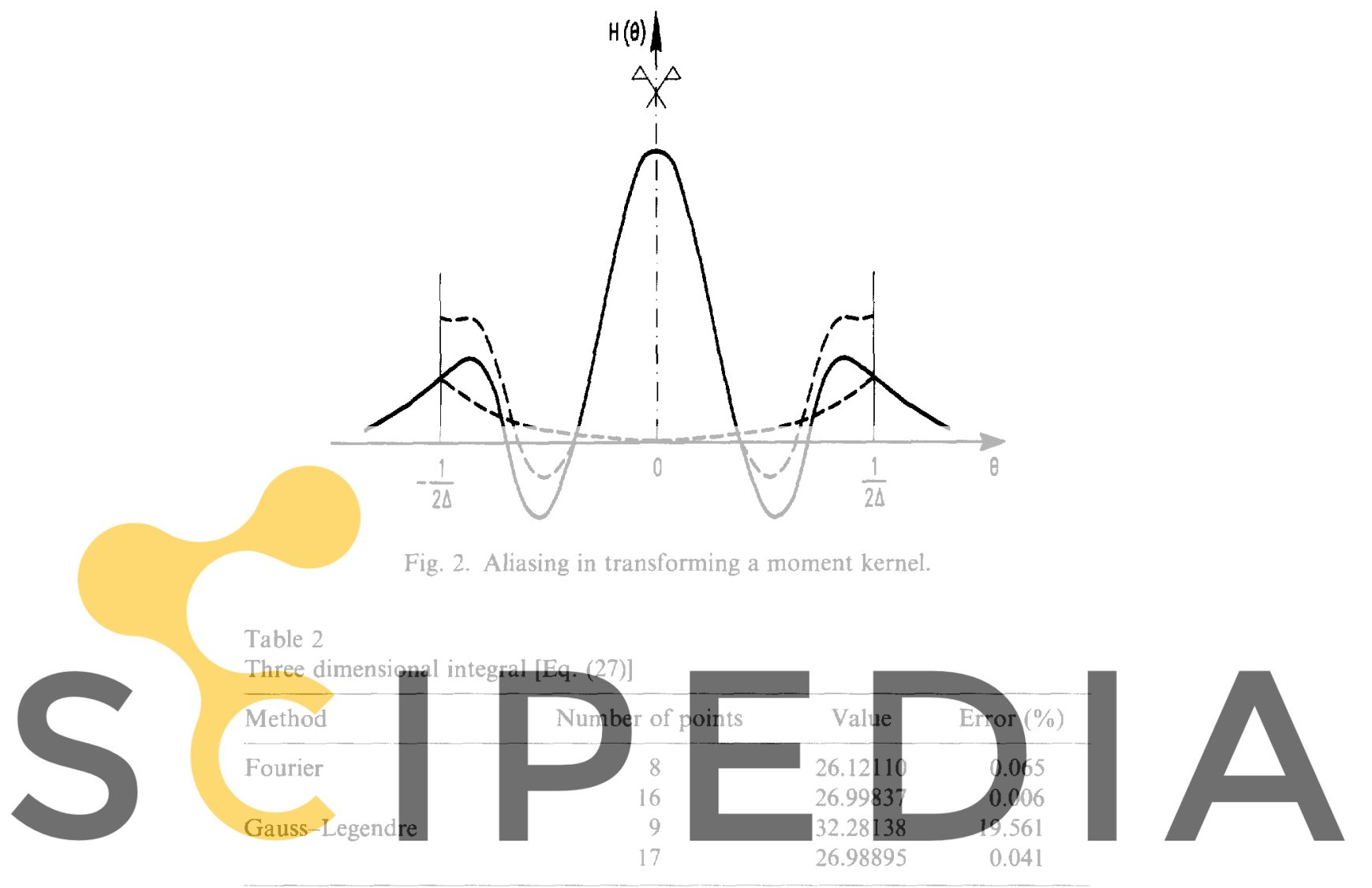

Register for free at https//www.scipedia.com to download the version without the watermark value than the Gauss-Legendre technique, due to the relative insensitivity of the zero frequency ordinate to aliasing mentioned above, and that its accuracy is better than that of the latter when using a similar number of points. Thus, the accumulated error for a calculation of several seconds of response (which is important for reliability estimations at the tails of the density function) will be less when using the present approach.

The algorithm was also compared with Simpson and Romberg methods. In general, the Fourier approach showed to be about one order of magnitude more accurate than the three conventional techniques studied. The reason for such superior accuracy of the Fourier method lies in the fact that while the transform is linked to the integrand by a mathematical relationship, Gaussian quadratures give exact results only for integrands that can be expressed as a polynomial multiplied by the weighting function, which is not the case in the present context. On the other hand, Simpson or similar rules are exact only for polynomial integrands of specific order.

\section{Numerical examples}

In order to test the numerial advantages of the proposed algorithm some numerical cases corresponding to well known highly nonlinear oscillators were studied. They are the Van der Pol, 
quadratic damping and Bouc-Wen hysteretic systems, which were excited by Gaussian white noise. Note that the last two have no direct solution by closure schemes. The aim was to obtain the stationary density function of the displacement in the first two cases and the non stationary evolution of the joint density of $X$ and $Z$ in the third case. In all cases the computation was carried out up to a time of $5 \mathrm{~s}$, using a time step of $0.001 \mathrm{~s}$ and 16 points per axis of integration. The initial covariance matrix was built with diagonal values equal to 0.04 and zero off diagonal entries. The maximum order of equations generated in all cases $K=4$. No numerical difficulties were found in these analyses. All results are compared with Monte Carlo simulation $(50,000$ samples).

\subsection{Van der Pol oscillator}

\section{The equation of motion of this case is}

$$
\dot{X}(t)+0.2\left(-1+10 X^{2}(t)\right) \dot{X}(t)+X(t)=W(t)
$$

where the excitation is a white noise with autocorrelation function $R(\tau)=\delta(\tau)$. The results are shown in Fig. 3.
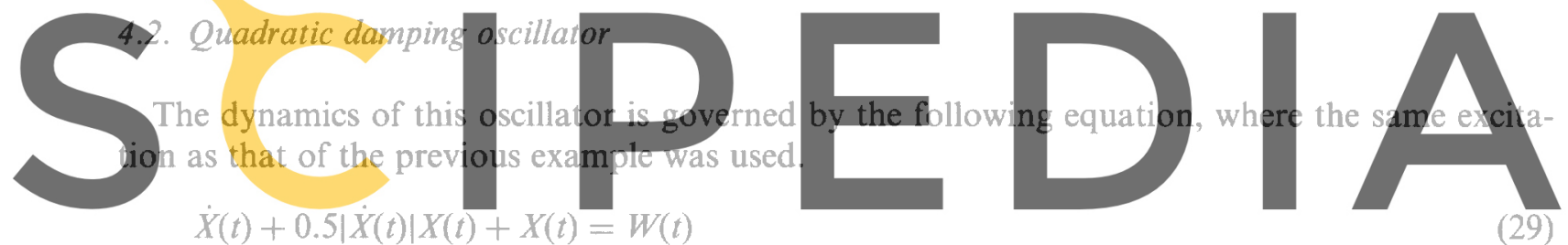

Register for free at https//www.scipedia.com to download the version without the watermark As Fig. 4 shows the agreement with Monte Carlo simulation is again excellent.

\subsection{Bouc-Wen hysteretic oscillator}

Unlike the previous cases, which imply the calculation of bidimensional integrals, this example requires $3 \mathrm{D}$ integration due to the need of adding to the equation of motion

$$
\dot{X}(t)+0.2 \omega \dot{X}(t)+\alpha \omega^{2} X(t)+(1-\alpha) \omega^{2} Z(t)=-W(t)
$$

the differential equation for the auxiliary variable $Z(t)$

$$
\dot{Z} A \dot{X}(t)-\beta|\dot{X}(t)||Z(t)|^{n-1} Z(t)-\gamma \dot{X}(t)|Z(t)|^{n}
$$

The parameters have been set at $\omega=2 \pi / 3, \alpha=0.1, A=1, \beta=\gamma=0.5$ and $n=1$. The noise intensity was set at $S_{0}=1$, which was intended to produce a strong nonlinear behaviour. Figs $5(\mathrm{a})-(\mathrm{c})$ display the evolution of the joint density function of $(X, Z)$. It can be seen that in the first states the system responses almost linearly, as indicated by the similarity of the joint function with the Gaussian density. In later instants, however, the large departure from Gaussianity and linearity is 


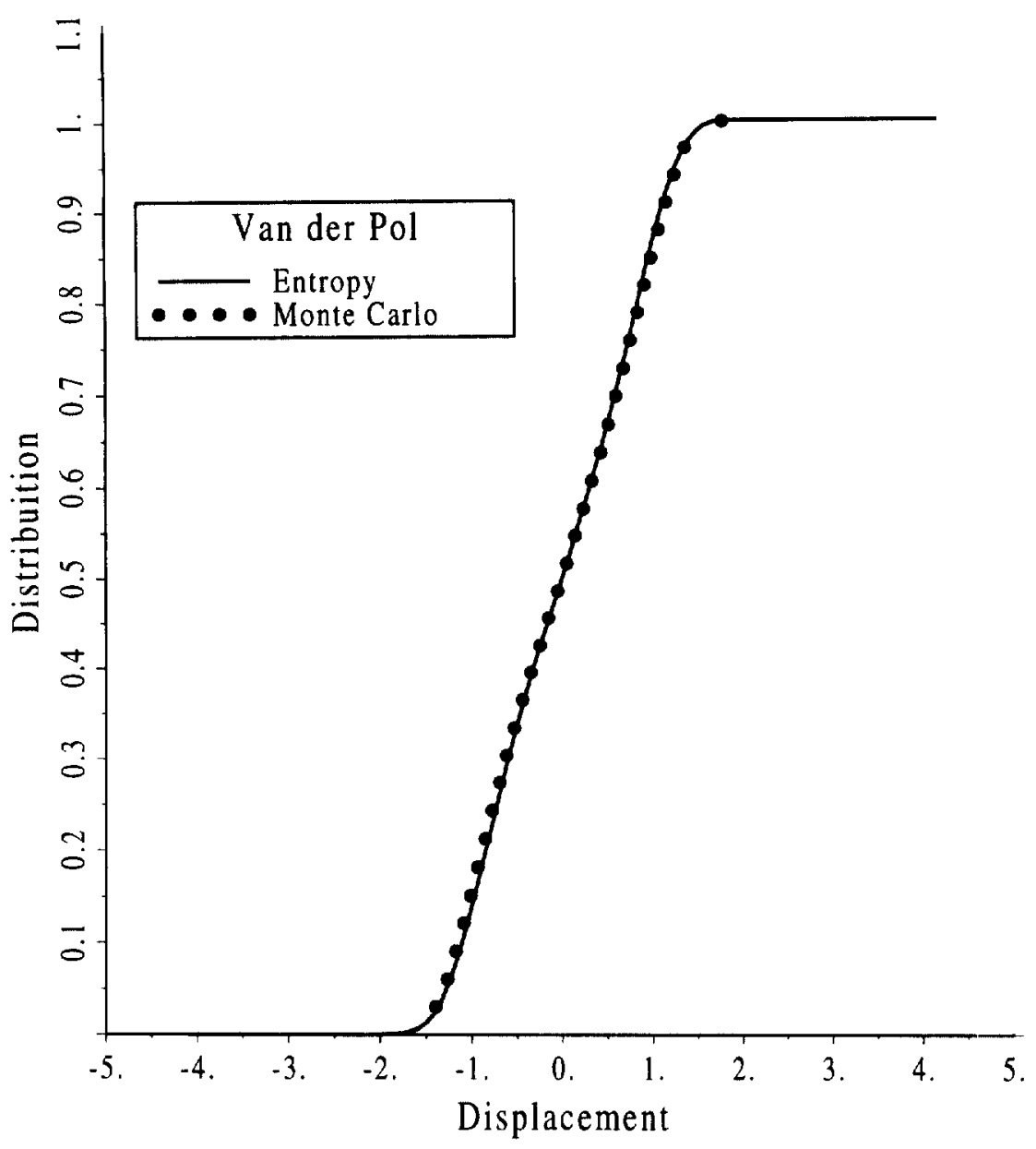

Fig. 3. Displacement stationary distribution of the Van der Pol oscillator.

evidenced by the increasing bimodality on the variable $Z$, which represents the restoring force [1]. In fact, as the dynamics of the system evolves, there is an increasing concentration of $Z$ near its maximum, which is given by

$$
\max (Z)=\left(\frac{A}{\beta+\gamma}\right)^{\frac{1}{n}}
$$

It equals one in this case.

Attempts to solve these examples were also performed using Gauss-Legendre integration. The system of algebraic Eq. (18) was solved by singular value decomposition. The calculation in all cases collapsed at its beginnings by overflow when using single or double precision. In the later case the failure time instants were 1.67, 0.95 and $1.31 \mathrm{~s}$ for the three examples considered, respectively, so that in not a case the system reached stationarity. Several attempts were made 


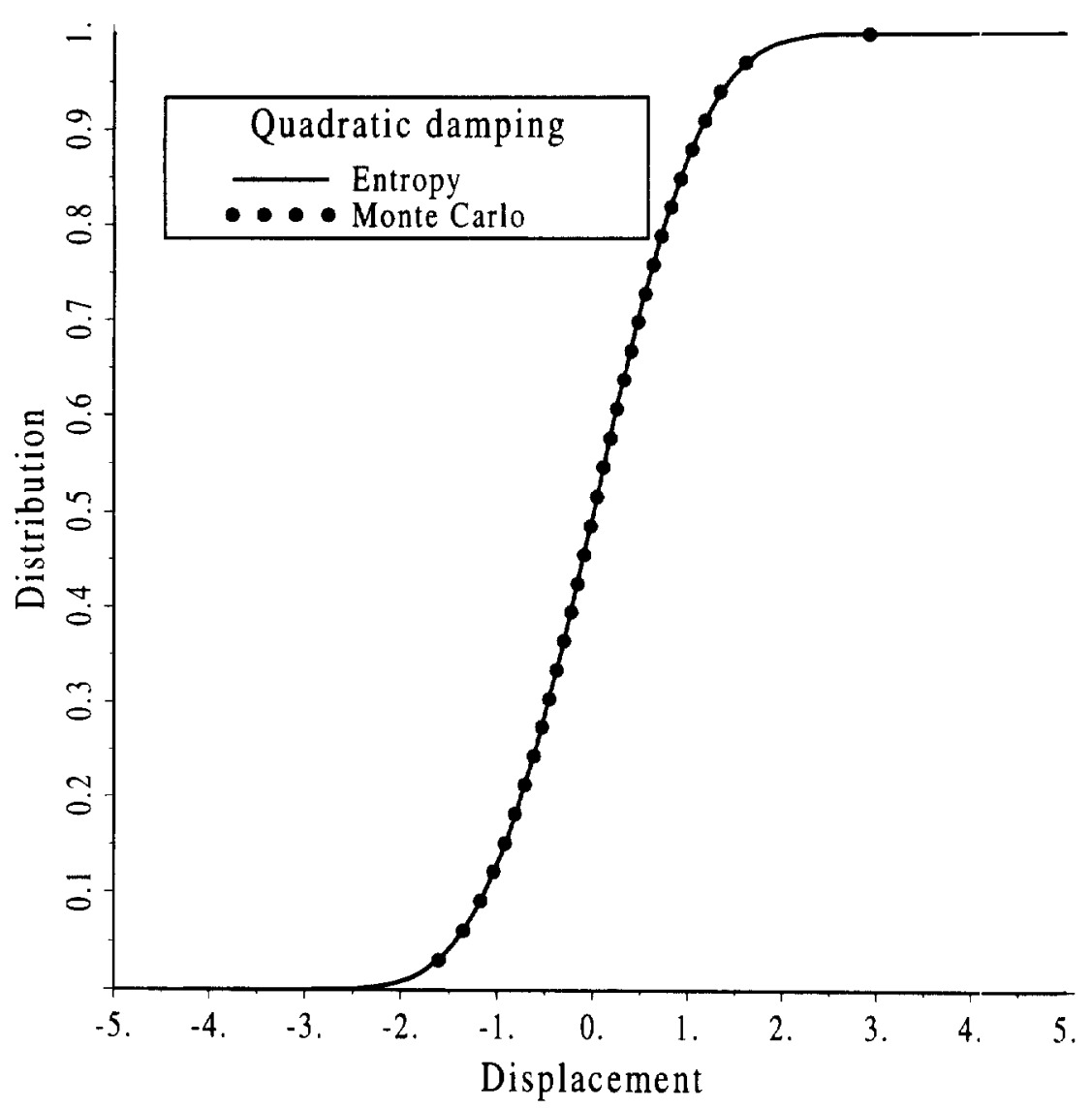

Fig. 4. Displacement stationary distribution of the quadratic damping oscillator.

using a higher number of quadrature points and/or different strategies for solving the linear system of equations without success. The same situation was faced when calculating the response of other oscillators, such as Duffing, Coulomb, etc.

There are at least two numerical reasons for explaining such breakdowns. The first is the location of the dicretization points for integration. In the Gauss-Legendre technique they are unevenly spaced, being the closer to each other, the farther their distance from the origin. Thus, when evaluating the density function (4) and the moment kernels (2), in which the values of the state variables are raised to high powers, the argument of the integrands can reach very large values at some instants, specially in the first case due to the implied summation. The even discretization of the Fourier technique prevents this from ocurring. But the most important cause for the failures is by far the ill-conditioning of matrix $A$ in Eq. (18), as measured by its singular values. It was observed that the ratio of the extreme singular values suddenly jumped beyond the machine precision when using the Gauss-Legendre technique and that, if the Lagrange parameters associated with the lowest singular values were removed from the equations, the only effect was to postpone the numerical collapse to some later instant. In contrast, when using the 
(a) $f(x, y)$

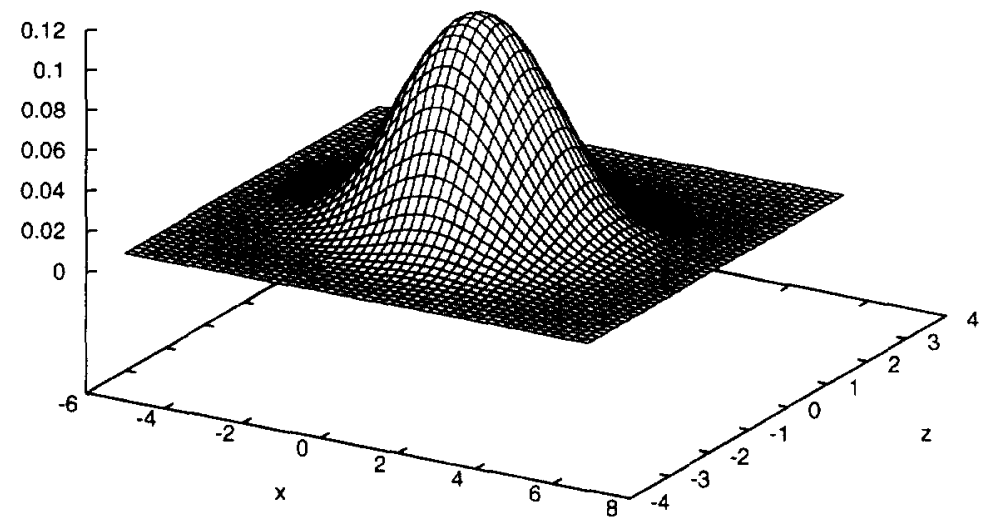

(b) $f(x, z)$

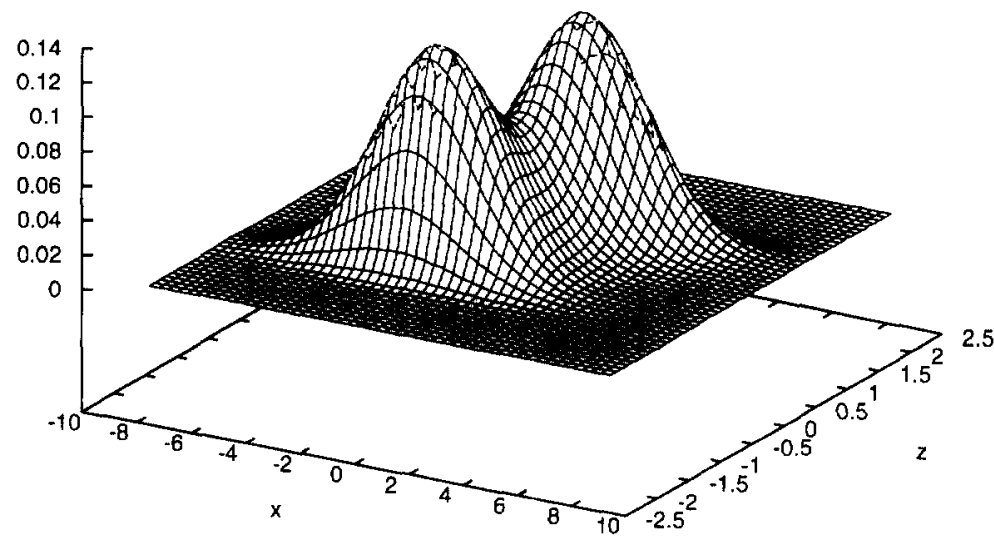

(c) $f(x, z)$

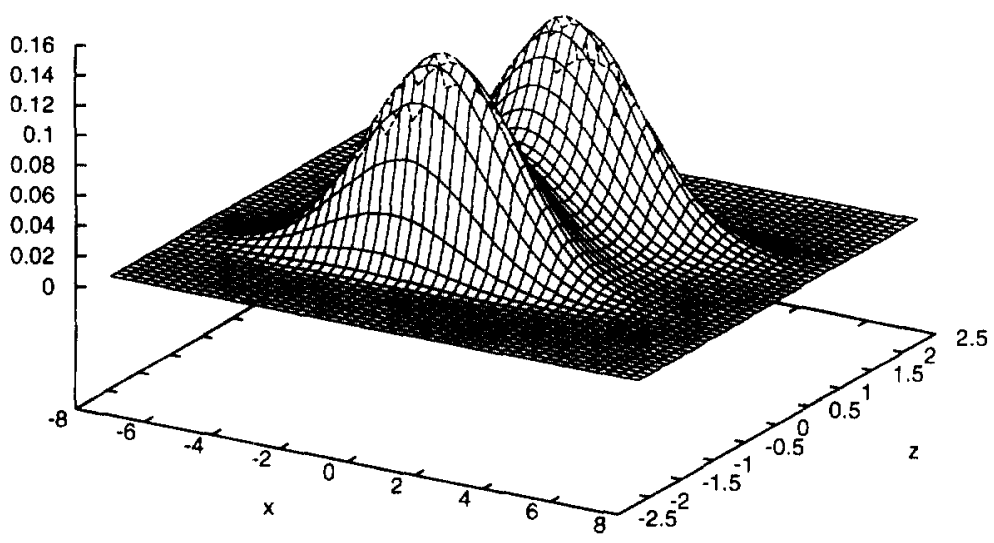

Fig. 5. Joint density of displacement and hysteretic function. (a) $t=0.5 \mathrm{~s} ;$ (b) $t=1.0 \mathrm{~s}$; (c) $t=5.0 \mathrm{~s}$. 
Fourier approach, the ratio of the maximum to the minimum singular value was always observed to be much less than in the Gauss-Legendre case. Since there is no direct mathematical link between precision of the integration and the ill-conditioning of the matrix, the superiority of the Fourier approach can only be sated here on the empirical basis of the many examples analysed, three of which are exposed here. Finally, it is important to note that the computational time required by the proposed Fourier algorithm per s of response analysis was similar to that required by the Gauss-Legendre quadratures.

\section{Conclusions}

A algorithm for calculating the large number of multidimensional integrals required by the method of maximum entropy when applied to stochastic dynamics problems has been presented. It is based on the Fourier-type relationship linking statistical moments and the characteristic function. By calculating only the zero frequency point of the multidimensional Fourier transform and taking advantage of its properties the algorithm leads to a method that is as economical as the use of Gauss-Legendre quadratures but more accurate and numerically more stable than it. By means of the analysis of some non linear oscillators it has been shown that the non stationary response can be obtained without any difficulty. This makes the method of maximum entropy more useful for the analysis of larger dynamical systems.

\section{Acknowledgements}

Financial supports for the realization of the present research has been received from the Colombian Science Institute Colciencias and the National University of Colombia. The work has also been partially supported by the ESPRIT programme of the Comision of European Countries, project PROMENVIR, ESPRIT 20189, and by the "Dirección General de Investigación Científica y Técnica" (DGICYT) of the Spanish Government under the grant no. PB93-1040. The support is gratefully acknowledged.

\section{References}

[1] Roberts JB, Spanos PD. Random vibration and statistical linearization. Chichester, UK: John Wiley, 1990.

[2] Muscolino G. Response of linear and non-linear structural systems under Gaussian or non-Gaussian filtered input. In: Casciati F, editor. Dynamic motion. Chaotic and Stochastic Behaviour. Wien: Springer-Verlag, 1993.

[3] Nielsen SRK, Mørk KJ, Thoft-Christensen P. Stochastic response of hysteretic sysitems. Structural Safety 1990;9:59-71.

[4] DiPaola M, Ricciardi G, Vasta M. A method for the probabilistic analysis of nonlinear systems. Probabilistic Engineering Mechanics 1995;10:1-10.

[5] Langtangen HP. A general numerical solution method for the Fokker-Planck equation with applications to structural reliability. Probabilistic Engineering Mechanics 1991;6:33 48.

[6] Spencer BF, Bergman LA. On the numerical solution of the Fokker-Planck equation for nonlinear stochastic systems. Nonlinear Dynamics 1993;4:357-72. 
[7] Shiau LC, Wu TY. A finite element method for analysis of a non-linear system under stochastic parametric and external excitation. International Journal of Nonlinear Mechanics 1996;31:193-201.

[8] Trębicki J, Sobczyk K. Maximum entropy principle and non stationary distributions of stochastic systems. Probabilistic Engineering Mechanics 1996;11:169-78.

[9] Sobczyk K, Trębicki J. Maximum entropy principle in stochastic dynamics. Probabilistic Engineering Mechanics 1990;5:102-10.

[10] Kapur JN. Maximum entropy models in science and engineering. New Delhi: John Wiley and Sons, 1989.

[11] Gzyl H. The method of maximum entropy. Singapore: World Scientific, 1995.

[12] Øksendal B. Stochastic differential equations. Berlin: Springer-Verlag, 1995.

[13] Kloeden PE, Platten E. Numerical solution of stochastic differential equations. Berlin: Springer-Verlag, 1992.

[14] Oppenheim AV, Schafer RW. Discrete-time signal processing. New Jersey: Prentice Hall International, 1989.

[15] Sobczyk K. Probability distributions for stochastic systems and maximum entropy method. In: Schuëller GI, editor. IASSAR Report on Computational Stochastic Mechanics. Probabilistic Engineering Mechanics 1997;12:266-69.

[16] Press WH, Teukolsky SA, Vetterling WT, Flannery BP. Numerical recipes in FORTRAN. New York: Cambridge University Press, 1992.

[17] Roy RV, Spanos PD. Padé-type approach to nonlinear random vibration analysis. In: Lin YK, Elishakoff I, editors. Stochastic structural dynamics 1. Berlin: Springer-Verlag, 1991. 